\title{
IMU sensor-based data glove for finger joint measurement
}

\author{
Muhammad Ajwad Wa'ie Hazman ${ }^{1}$, Ili Najaa Aimi Mohd Nordin², Faridah Hanim Mohd Noh', \\ Nurulaqilla Khamis ${ }^{4}$, M. R. M. Razif ${ }^{5}$, Ahmad Athif Faudzi ${ }^{6}$, Asyikin Sasha Mohd Hanif ${ }^{7}$ \\ ${ }^{1,2,3,4}$ Department of Electrical Engineering Technology, Faculty of Engineering Technology, \\ Universiti Tun Hussein Onn Malaysia (UTHM), Malaysia \\ ${ }^{2,5}$ Cybernetics Research Group (CRG), Faculty of Engineering Technology, \\ Universiti Tun Hussein Onn Malaysia (UTHM), Malaysia \\ ${ }^{4,7}$ Centre for Artificial Intelligence and Robotics, Malaysia-Japan International Institute of Technology, \\ Universiti Teknologi Malaysia, Malaysia \\ ${ }^{6}$ School of Electrical Engineering, Faculty of Engineering, Universiti Teknologi Malaysia, Malaysia
}

\section{Article Info}

Article history:

Received Feb 4, 2020

Revised Apr 6, 2020

Accepted Apr 20, 2020

\section{Keywords:}

Data glove

Finger flexion

Finger joint measurement

Flexible bend sensor

Inertial measurement unit

\begin{abstract}
The methods used to quantify finger range of motion significantly influence how hand disability is reported. To date, the accuracy of sensors being utilized in data gloves from the literature has been ascertained yet need further analysis. This paper presents a sensor-based data glove for finger joint measurement developed for collecting a range of motion data of distal interphalangeal, proximal interphalangeal and metacarpophalangeal finger joints of an index finger. In this study, three inertial measurement sensors and two flexible bend sensors were attached to the finger joint points on the glove to detect angle displacement. The angle displacements were acquired using Arduino and MATLAB software interface. Goniometry was used to allow accurate comparative measurement. Low percentage of error resulted from inertial measurement unit ( $0.81 \%$ to $5.41 \%$ ), indicates high accuracy. On the other hand, flexible bend sensor shows low accuracy $(11.11 \%$ to $19.35 \%$ error). In conclusion, the inertial measurement unit sensor, MPU6050 can be a reliable solution for tracking the progress of finger rehabilitation exercises. In order to motivate patients to adhere to the therapy exercises, interactive rehabilitation game will be developed in the future incorporating MPU-6050 sensors on all five fingers.
\end{abstract}

Copyright () 2020 Institute of Advanced Engineering and Science. All rights reserved.

\section{Corresponding Author:}

Ili Najaa Aimi Mohd Nordin,

Cybernetics Research Group, Faculty of Engineering Technology,

Universiti Tun Hussein Onn Malaysia (UTHM),

Jalan Panchor, 84600 Pagoh, Johor, Malaysia.

Email: ilinajaa@uthm.edu.my

\section{INTRODUCTION}

In most countries, stroke is the second most common cause of death and one of the main causes of adult disability. Although 800000 individuals each year that have been afflicted with stroke struggle with daily tasks, more than two thirds of stroke patients receive rehabilitation services after hospitalization [1]. In medical and rehabilitation practice, a system that can monitor the patient's rehabilitation treatment and progress as one of the assessment frameworks is vital for evaluating body function [2].

Finger joints and limbs are body parts that have range of motion (ROM). A measurement of finger joint ROM is often a crucial component of a physical evaluation since the hand function becomes drastically reduced in the absence of joint motion. Finger ROM is measurable and frequently being assessed by physiotherapist using traditional goniometry measurement method. Finger ROM assessment requires accurate monitoring to evaluate the effectiveness of the rehabilitation treatments and to precisely regulate the rehabilitation process of stroke patient [3]. A finger joints measurement can be complicated due to the large 
number of joints in a small space, the complex interrelated movement from tendons of two joint muscles, and the short axial segments of the bone [4]. Sometimes the presence of edema, massive scars, enlarged and malformed joints, the difficulty of stabilization and force on the bony segments, immature wounds, and extreme sensitivity of the severely injured hand can be a compounded that create a problem in measurement. To overcome these problems of measuring that fulfill the objective of the assessment, the goniometer provides both reliability and adaptability [5].

The demand for rehabilitation has increased annually, generally in accordance to the increase of public spending. This has motivated the demand of providing mobile and accurate rehabilitation robotic system for speeding up the recovering process. In this regard, robotic systems that offers practicality were developed [6-8]. Most of the rehabilitation will focus on the identification and resolution of movement problems to track the range of movement abnormalities for each individual patient so the outcome of the rehabilitation can be improved when the feedback of their movement is obtained. Typical camera motion sensor analysis system used for rehabilitation consists of multiple wearable sensors, for example, passive/active optical markers, EMG/EEG/ECG, inertial sensors, or force plates. In previous studies [9-12], non-contact-based measurement system such as cameras or depth-based camera to capture the ROM were inconvenient for mobile rehabilitation as a camera should be placed in a room with specific lighting condition and environment. Camera-based motion capture analysis for finger joint measurement requires large laboratory and space for data collection, yet costly and not portable, so this is impractical for flexible, mobile clinical and home-use rehabilitation.

VICON is one of the optical motion analysis systems that is of high interest to researchers; however, current marker-based and marker less, single or multiple infrared or RGB camera motion analysis systems have restrictions. For example, this system depends on the color of the fabric, is a time-consuming process, and the systems lack portability yet costly [12].

A large number of data glove for providing information about finger flexion have been proposed in recent years [2, 3, 10-22]. A data glove is consisted of a system with sensors, an electronics microcontroller for data acquisition / processing and power supply. With the sensor attached, the data glove can be worn on the user to evaluate the ROM of the finger. The data glove allows each finger to have joints ROM measurement. The glove is equipped with 22 resistive sensors that can measure the displacement of a finger [13, 18-25], and can communicate with the personal computer (PC) through wireless or USB serial connection.

Flexible bend sensor is one of resistive sensor used to measure and evaluate finger joints movements [24]. This system is lightweight and is not costly which can be developed with only a few dollars. However, the system lacks precision due to the inelasticity of plastic component of the sensor which shows difficulties in reverting back to the initial position after being flexed for many times. Although the flex bend sensorbased data glove in [17] is lightweight and thin, it could result in inaccurate assessments results. But with an advancement in semiconductor technology, some studies have applied inertial measurement units (IMUs) sensor to measure the hand or finger joint ROM [2, 3, 14-16] and spine posture [26]. Some of the data gloves are using a 6-axis IMU sensors [15], some with a 9-axis IMU sensor [3, 14, 27]. The 9-axis IMU sensor consists of 3-axis accelerometer, 3-axis gyroscopes, and 3-axis magnetometers that can produce output of acceleration, angular velocity and magnetic field, while most of the previous data gloves used a 9-axis IMU sensor that requires a specific MCU to process the data, for example MSP430F5438A. Both flexible bend sensor and IMU are lightweight, low cost and easily accessible.

PC monitoring mechanism-based LabView software can provide the graphical user interface (GUI) of sensor monitoring. The GUI's functionality is to monitor the work principal as well as the system's ongoing data transfer and data acquisition. The GUI system is connected via the Arduino microcontroller to the exoskeleton system. Consequently, the system input from the EMG sensors and IMU sensors can be monitored in much simpler GUI data rather than the serial monitoring mechanism data. The monitoring system is capable of monitoring both hand postures from the reading of the IMU sensor with the angular motion display. The IMU angular motion data determines the positional structure flow of the hand's graphical display as the mechanism indicates the hand's elevation level from the initial position. In addition, the number of rehabilitation repetition will also be recorded when the arm moves [28].

In addition, an online GUI monitoring system was designed through the LabView software and was able to display the exact value. Online monitoring is designed to enable doctors to monitor the performance and development of their patients. The online monitoring system can also control the programmable flow; start the stimulation or even stop the simulation. The online monitoring system was carried out in accordance with the research's objectives. However, concerning the connectivity between the Arduino and the LabView, the system experiences several minor lags of receiving data. It took approximately 3 seconds to display or refresh the data with new data tabulation. Sometimes the delay factor is dependent on the internet connectivity from the domain user to the host [28]. 
In this paper, 6-axis IMUs and 2.2-inch flexible bend sensor are used to acquire finger joint ROM using Arduino microcontroller. A more easily accessible MATLAB software for developing a GUI and plotting ROM data measurement is also utilized. A comparison and analysis of two different sensor-based sensing methods for compromising a better measurement method for finger ROM are also studied.

\section{METHODOLOGY}

\subsection{Prototype development}

The purpose of developing the data glove is to measure the ROM of finger joint and compare the reliability of two types of displacement sensors. Three of the 6-axis IMU sensors were attached on the data glove to assess each finger joint MCP, DIP, PIP ROM and two of 2.2-inch flexible bend sensor were fixed in MCp and DIP joints for rehabilitation monitoring purposes. A GUI on MATLAB was developed to monitor the ROM of every finger joint for data analysis. The results obtain were compared with goniometer data.

Figure 1 shows the block diagram of the Data Glove system. The data glove consists of three 6-axis IMU sensors and two 2.2-inch flexible bend sensors to record the displacement of the finger joints. The data from both sensors were sent to the Arduino Nano ATMega328 for translating the displacement into ROM angle data. Simultaneously, the ROM data with $\mathrm{X}, \mathrm{Y}$ and $\mathrm{Z}$ axis position of the MCP, DIP, PIP joints were sent to the MATLAB GUI via serial UART connection for monitoring from a laptop.

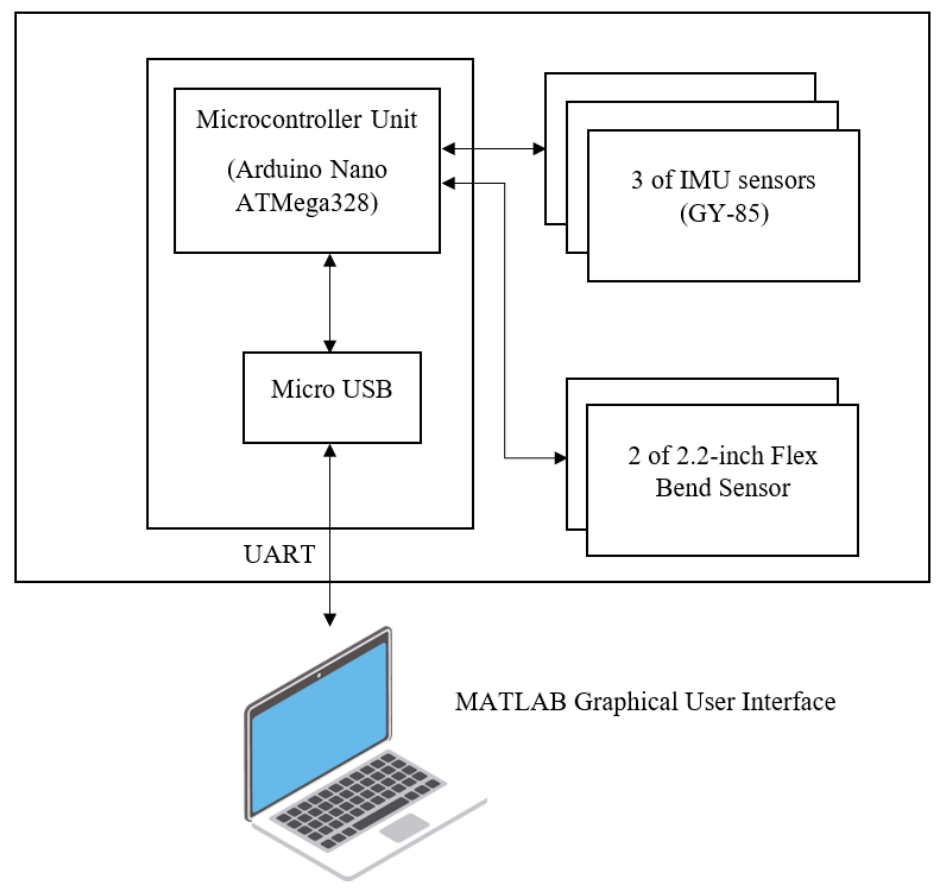

Figure 1. Block diagram

A cloth material glove with one button to secure the glove on hand is used. The electronic sensors were secured on the glove by sewing to ensure the components are locked at joints point while subjects move their hand. First, the two-flex bend sensor were secured only on MCP ad PIP joints points. The 2.2-inch flex bend sensor will not fit the length of distal part of finger, thus, DIP bending angle will not be measured using this sensor. Flexible bend sensor is a resistive carbon-based sensor which operates based on varying the resistance value correlated to the bend radius. Then, three IMU sensors were fixed on top of the flex sensor's position at MCP, DIP and PIP points of finger joint. The electronics circuit consists of the Arduino microcontroller, $100 \mathrm{~K}$ resistor and pinboard for the input of the IMU-sensor were soldered on the donut board. The donut board was sewed on the dorsal side of the glove. Figures 2 and 3 show the developed prototype of the IMU sensor-based data glove for finger joint measurement. 

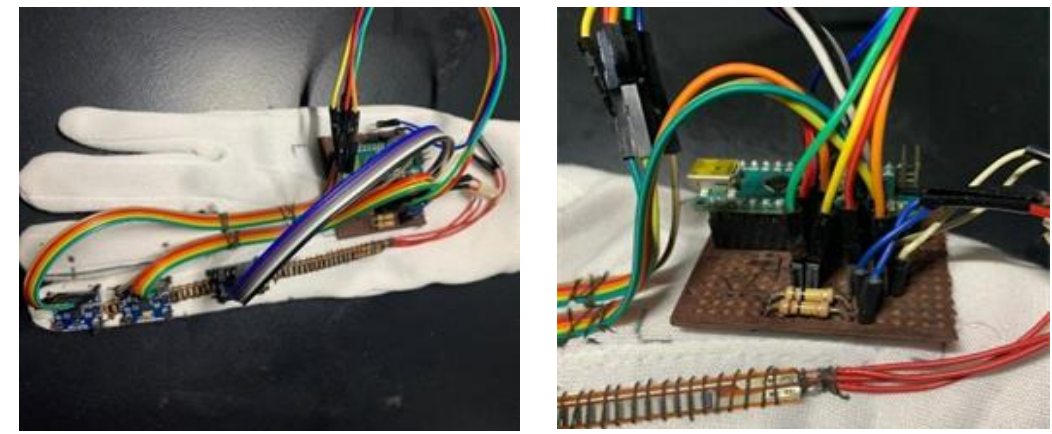

Figure 2. Top view of data glove

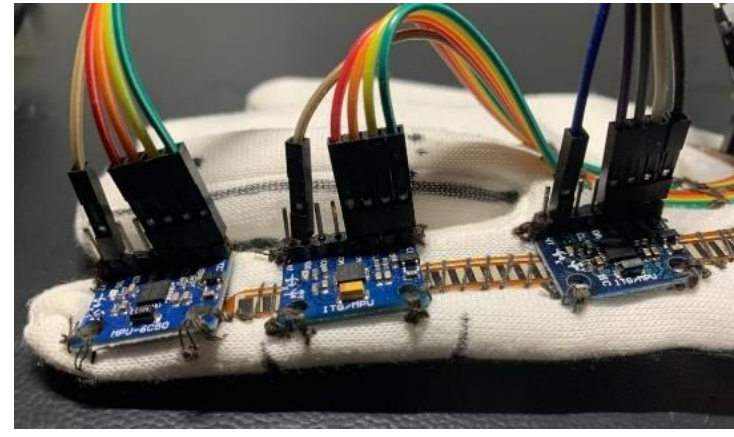

Figure 3. Position of IMU sensor and flex bend sensor

\subsection{Data measurement setup}

Goniometry is used as a reference for validating the accuracy of the ROM data measured from the IMUs and flex bend sensors. The ROM of MCP, PIP and DIP finger joints were measured from ten male healthy subjects from Universiti Tun Hussein Onn Malaysia. The subjects that can fit almost perfectly to the $\mathrm{M}$ size data glove were selected. Initially, the subjects were instructed to simulate paralyze condition, similar to relaxed state as shown in Figure 4. Figures 5, 6 and 7 shows the definition of MCP, PIP and DIP flexion angle.

Note that the angle when the hand is in relaxed position is not exactly zero degree. Before the electronics sensors are being tested for flexion motion, the angle of the sensors when in relaxed state is recorded. The exact flexion angle value of each finger joint is obtained by substracting the relaxed state angle from fully flexed state angle. The relaxed state angle was programmed into the Arduino microcontroller before each measurement starts. The data were collected three times for each individual and the average values are tabulated.

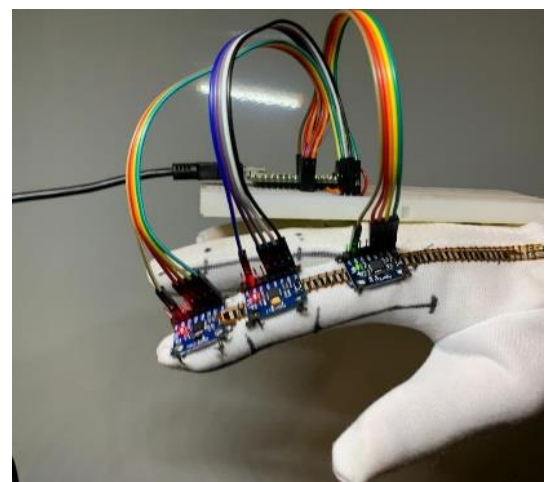

Figure 4. IMU sensor-based data glove when the finger is in relax position

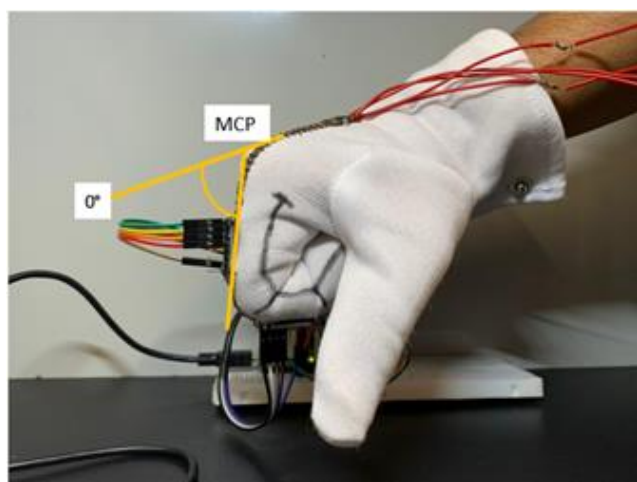

Figure 5. The angle for MCP when fully flexed 


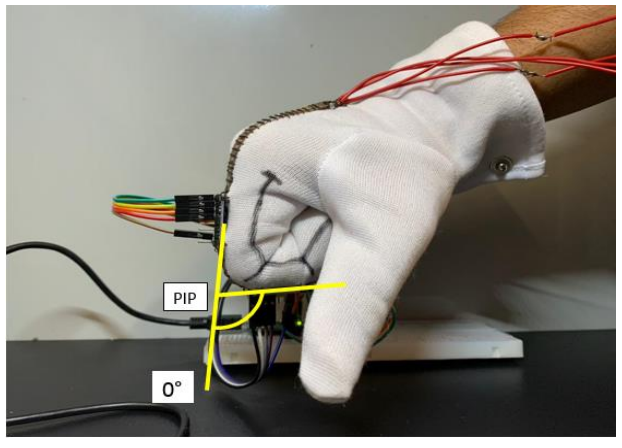

Figure 6. The angle of PIP when fully flexed

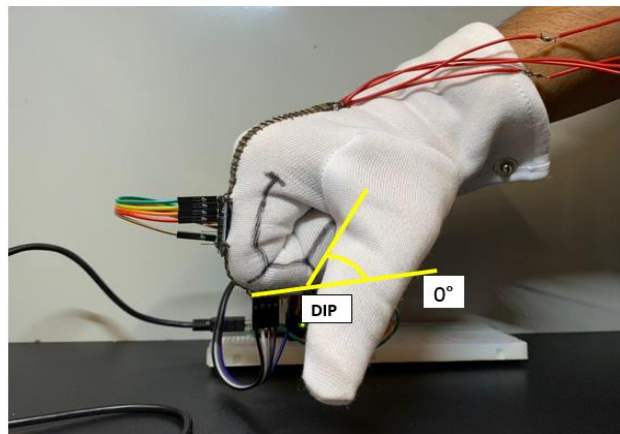

Figure 7. The angle of DIP when fully flexed

\section{RESULTS AND ANALYSIS}

This research attempts to obtain the accuracy of electronics sensors that are widely used to measure the ROM of finger joints. The measurement was taken when the hand is in relaxed and fully flexed conditions. The result from the IMU sensor and flex bend sensor were compared with that of goniometer. The graph and table in Figure 8 illustrate the measurement results. For full flexed condition of DIP finger joint, goniometer measurement is $43^{\circ}$ while IMU sensor is $45.33^{\circ}$. Flexible bend sensor was not installed to measure the DIP finger angle because it cannot be nicely fit on the finger. The sensor is longer than the finger when the centre point of the bend sensor is being fixed at the point of DIP joint. For full flexed condition of PIP finger joint, goniometer measured $62^{\circ}$, while flex bend sensor obtained $74^{\circ}$ and IMU sensor obtain $62.5^{\circ}$. Lastly, when MCP finger joint is fully flexed, the goniometer measured $63^{\circ}$, while flex bend sensor obtained $70^{\circ}$ and IMU sensor obtained $66^{\circ}$. As can be seen from Figure 8, IMU sensor shows the nearest measurement to the ideal value from the goniometry. The percentage of error using electronics sensors were calculated using Formula 1 and 2. The results are tabulated in Table 1.

$$
\begin{aligned}
& \text { Percentage of error }=\frac{I M U \text { sensor angle-Goniometer angle }}{\text { Goniometer angle }} \times 100 \% \\
& \text { Percentage of error }=\frac{\text { Flex bend sensor angle-Goniometer angle }}{\text { Goniometer angle }} \times 100 \%
\end{aligned}
$$

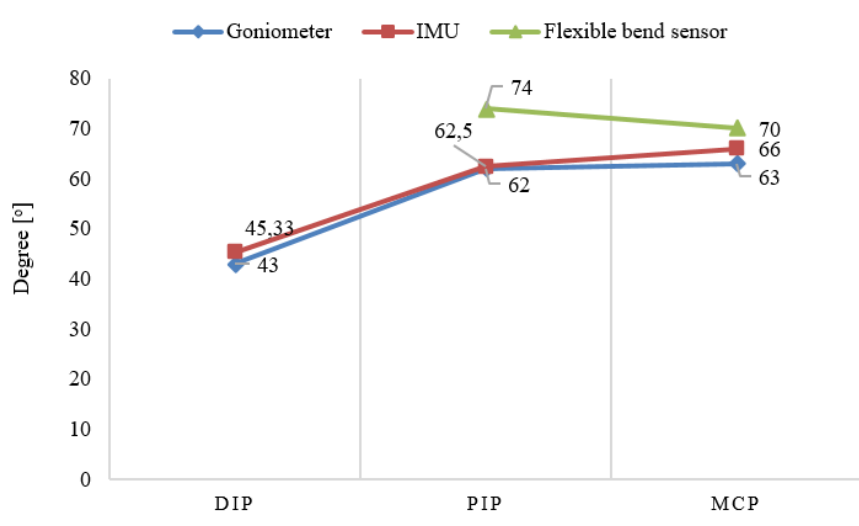

Table 1. Percentage error of IMU sensor compare with flex bend sensor

\begin{tabular}{ccc}
\hline Finger Joints & IMU sensor & Flex bend sensor \\
\hline DIP & $5.41 \%$ & - \\
PIP & $0.81 \%$ & $19.35 \%$ \\
MCP & $4.76 \%$ & $11.11 \%$ \\
\hline
\end{tabular}

Figure 8. Finger ROM measurement from goniometry and electronic sensing methods

From the results, the flexible bend sensor exhibits higher percentage of error than IMU MPU-6050 sensor during both PIP $(19.35 \%>0.81 \%)$ and MCP angle measurement $(11.11 \%>4.76 \%)$. This shows that the MPU-6050 is more reliable that flexible bend sensor. Lower accuracy of flex bend sensor might due to the difficulties in reverting back to the initial position after being flexed for many times. The graphical user interface (GUI) of the monitoring system is also developed in MATLAB. Figure 9 and 10 show the PIP finger joint flexion angle and a 3D visualization of a rectangle object following finger joint movement. The 3D visualization is to ease physiotherapy process and patients to evaluate the full flexion of finger in real-time. 


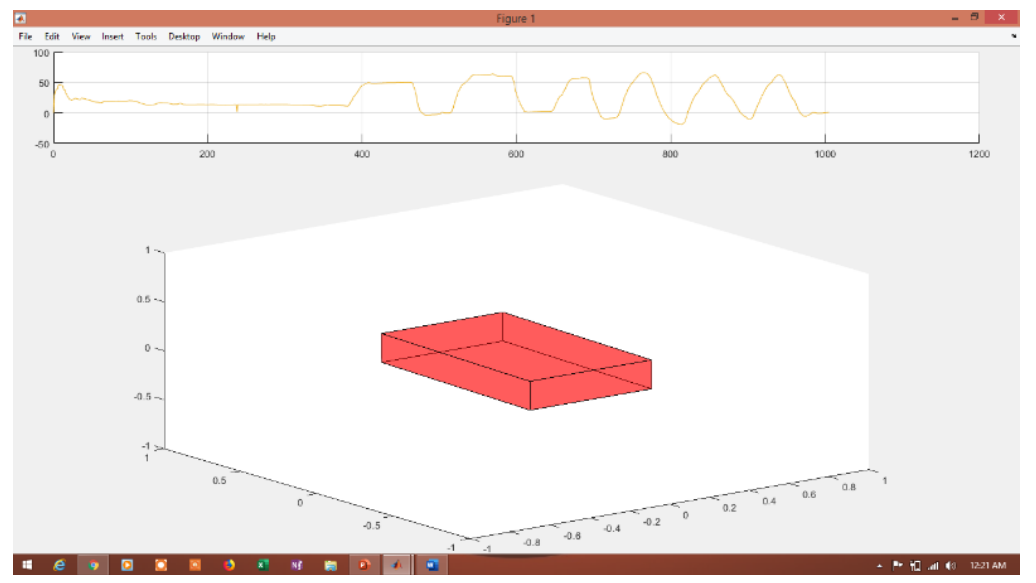

Figure 9. MATLAB GUI monitoring PIP angle from IMU sensor
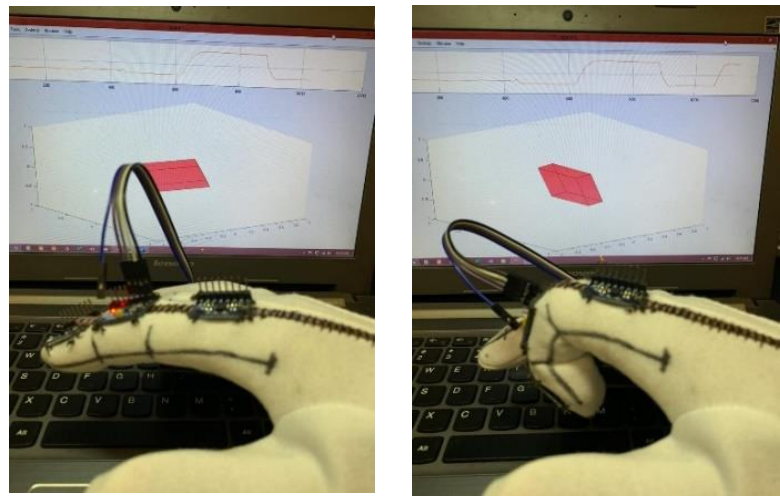

Figure 10. The simultaneous respond of rectangle object with the angle displacement of PIP finger joint

\section{CONCLUSION}

As a conclusion from the result obtained, IMU Sensor-based Data Glove can be implemented as an alternative tool for measuring and evaluating the finger joints ROM of stroke patients or people with finger disability. With the aid of GUI monitoring, rehabilitation process can be conducted efficiently. Moreover, the data from the monitoring system can be used to control a motor or actuator for a master-slave application. Several recommendations are proposed to enhance the accuracy of the current glove and the GUI monitoring system. A user-friendly and stretchable glove can be used, as a stiff glove will restrict the movement of the jumper wire that connect the sensors worn on the glove to the microcontroller which will affect the accuracy of the sensor reading. Other than that, the 3-D object displayed on the GUI that respond to the movement of the glove worn on the finger can be upgraded to a more interactive intervention. A ball shaped 3-D object can be designed to expand and shrink when the finger ROM is increased and decreased. The data glove can be practical and useful tool for physician and medical staff to evaluate patient's finger ROM.

\section{ACKNOWLEDGEMENTS}

This research is fully supported by the FRGS RACER (RACER/1/2019/TK04/UTHM//8) grant. The authors are fully acknowledged by the Ministry of Education Malaysia and Universiti Tun Hussein Onn Malaysia for the approved fund which makes this important research viable and effective.

\section{REFERENCES}

[1] C. J. Winstein et al., "Guidelines for Adult Stroke Rehabilitation and Recovery: A Guideline for Healthcare Professionals from the American Heart Association/American Stroke Association," Stroke, vol. 47, no. 6, pp. e98-e169, June 2016. 
[2] B. Lin, P. Hsiao, S. Yang, C. Su, and I. Lee, "Data Glove System Embedded with Inertial Measurement Units for Hand Function Evaluation in Stroke Patients," IEEE Trans. Neural Syst. Rehabil. Eng., vol. 25, no. 11, pp. 2204-2213, Nov. 2017.

[3] B.-S. Lin, I.-J. Lee, S.-Y. Yang, Y.-C. Lo, J. Lee, and Jean-Lon Chen, "Design of an Inertial-Sensor-Based Data Glove for Hand Function Evaluation," sensors, vol. 18, no. 1545, pp. 1-17, May 2018.

[4] Q. Zhan, C. Zhang, and Q. Xu, "Measurement and Description of Human Hand Movement," in MATEC Web of Conferences 114, 2017, vol. 04002, pp. 1-6.

[5] G. F. Hamilton and P. A. Lachenbruch, "Reliability of Goniometers in Assessing Finger Joint Angle," Phys. Ther., vol. 49, no. 5, pp. 465-469, May 1969.

[6] S. Z. A. S. Kamarul Bahrin and K. S. Mohamed Sahari, "Solenoid Actuated Five-Fingered Robotic Hand Design: Evaluating the Capability of Solenoid Actuators in Generating Basic Finger Motions," Indonesian Journal of Electrical Engineering and Computer Science, vol. 15, no. 3, pp. 1273-1281, Sept. 2019.

[7] I. N. A. M. Nordin, A. A. Mohd Faudzi, M. Z. Kamarudin, D. E. O. Dewi, T. Rehman, and M. R. M. Razif, "Grip Force Measurement of Soft- Actuated Finger Exoskeleton," Jurnal Teknologi, vol. 78, no. 6-13, pp. 25-30, March 2016.

[8] H. Zhang, A. S. Kumar, F. Chen, Y. H. Jerry, and M. Y. Wang, "Topology Optimized Multimaterial Soft Fingers for Applications on Grippers, Rehabilitation and Artificial Hands," IEEE/ASME Trans. Mechatronics, vol. 24, no. 1, pp. 120-131, Feb 2019.

[9] S. Alexanderson and C. O. Sullivan, "Robust Online Motion Capture Labeling of Finger Markers," in MIG '16: Proceedings of the 9th International Conference on Motion in Games, 2016, pp. 7-13.

[10] A. Muro-de-la-herran, B. Garcia-zapirain, and A. Mendez-zorrilla, "Gait Analysis Methods: An Overview of Wearable and Non-Wearable Systems, Highlighting Clinical Applications,” sensors, vol. 14, no. 2, pp. 3362-3394, Feb. 2014.

[11] F. Schlagenhauf, S. Sreeram, and W. Singhose, "Comparison of Kinect and Vicon Motion Capture of Upper-Body Joint Angle Tracking," in IEEE 14th International Conference on Control and Automation (ICCA), 2018, pp. 674-679.

[12] C. Yang, V. Stankovic, S. Member, L. Stankovic, S. Member, and A. Kerr, "A Depth Camera Motion Analysis Framework for Tele-rehabilitation: Motion Capture and Person-Centric Kinematics Analysis," IEEE J. Sel. Top. Signal Process., vol. 10, no. 5, pp. 877-887, April 2016.

[13] S. Ganeson, R. Ambar, M. Mahadi, and A. Jamil, "Design of a Low-Cost Instrumented Glove for Hand Rehabilitation Monitoring System," in 6th IEEE Int. Conf. Control Syst. Comput. Eng., no. November, 2016, pp. 189-192.

[14] P. Hsiao, S. Yang, B. Lin, I. Lee, and W. Chou, "Data Glove Embedded with 9 - Axis IMU and Force Sensing Sensors for Evaluation of Hand Function," in International Conference of the IEEE Engineering in Medicine and Biology Society $(E M B C), 2015$, pp. 4631-4634.

[15] B. Lin, I. Lee, P. Hsiao, S. Yang, and W. Chou, "Data Glove Embedded with 6-DOF Inertial Sensors for Hand Rehabilitation," in $10^{\text {th }}$ International Conference on Intelligent Information Hiding and Multimedia Signal Processing, 2014. pp. 25-28.

[16] C. K. Jha and A. L. Chakraborty, "A Fiber Bragg Grating Strain Sensor-based Glove to Accurately Measure the Bend Angle of the Finger Flexed at the Proximal Interphalangeal Joints," in 2018 IEEE SENSORS, 2018, pp. 1-4.

[17] L. Yu, D. Xiong, L. Guo, and J. Wang, "A Remote Quantitative Fugl-Meyer Assessment Framework for Stroke Patients Based on Wearable Sensor Networks," Comput. Methods Programs Biomed., vol. 128, no. 88, pp. 100-110, May 2016.

[18] L. Dipietro, A. M. Sabatini, S. Member, and P. Dario, "A Survey of Glove-Based Systems and Their Applications," IEEE Transactions on Systems, Man, and Cybernetics, Part C (Applications and Reviews), vol. 38, no. 4, pp. 461-482, July 2008.

[19] M. Borghetti, E. Sardini, and M. Serpelloni, "Sensorized Glove for Measuring Hand Finger Flexion for Rehabilitation Purposes," IEEE Trans. Instrumentattion Meas., vol. 62, no. 12, pp. 3308-3314, July 2013.

[20] R. Alexandre, "Physical Rehabilitation based on Smart Wearable and Virtual Reality Serious Game," in IEEE International Instrumentation and Measurement Technology Conference (I2MTC), 2019, pp. 1-6.

[21] S. Pan et al., "A Sensor Glove for the Interaction with a Nursing-Care Assistive Robot," in IEEE International Conference on Industrial Cyber Physical Systems (ICPS), 2019, pp. 405-410.

[22] A. Suresh, G. Udupa, and D. Gaba, "Design and Development of Soft Robotic Hand for Vertical Farming in Spacecraft," Int. J. Robot. Autom., vol. 9, no. 1, pp. 6-16, March 2020.

[23] G. Ponraj and H. Ren, "Sensor Fusion of Leap Motion Controller and Flex Sensors using Kalman Filter for Human Finger Tracking," IEEE Sensors Journal, vol. 18, no. 5, pp. 2042-2049, March 2018.

[24] G. Saggio et al., "Long Term Measurement of Human Joint Movements for Health Care and Rehabilitation Purposes," in International Conference on Wireless Communication, Vehicular Technology, Information Theory and Aerospace \& Electronic Systems Technology, 2009, pp. 674-678.

[25] E. Abana, K. H. Bulauitan, R. K. Vicente, M. Rafael, and J. B. Flores, "Electronic Glove : A Teaching AID for the Hearing Impaired," International Jounal of Electrical and Computer Engineering (IJECE), vol. 8, no. 4, pp. 2290-2298, Aug. 2018.

[26] K. S. Tee, E. Low, W. Tony, S. Binti, M. Khialdin, and J. Bin, "A Posture Monitoring System with IMU For Ophthalmologist While Operating the Slit Lamp," Indonesian Journal of Electrical Engineering and Computer Science, vol. 18, no. 3, pp. 1262-1269, June 2020.

[27] C. Maremmani et al., "Preliminary Evaluation of Sensorfoot V1 And Senshand V1 In Assessing Motor Skills Performance of Parkinson's Disease Patients," J. Neurol. Sci., vol. 333, no. Supplement 1, p. e67, Oct. 2013.

[28] Y. Ganesan, S. Gobee, and V. Durairajah, "Development of an Upper Limb Exoskeleton for Rehabilitation with Feedback from EMG and IMU Sensor," Procedia - Procedia Comput. Sci., vol. 76, no. Iris, pp. 53-59, Dec. 2015. 